\title{
Thermal expansion and polarisation of (1-x)PNN-xPT solid solutions
}

\author{
M. V. Gorev ${ }^{\mathrm{a}, \mathrm{b}}$, I. N. Flerov ${ }^{\mathrm{a}, \mathrm{b}}$, K. Bormanis ${ }^{\mathrm{c}}$, A. Kalvane ${ }^{\mathrm{c}}$ \\ ${ }^{a}$ Kirensky Institute of Physics, Federal Research Center KSC SB RAS, Krasnoyarsk, Russia; \\ ${ }^{\mathrm{b}}$ Siberian Federal University, Krasnoyarsk, Russia; \\ ${ }^{\mathrm{c}}$ Institute of Solid State Physics, University of Latvia, Riga, LV-1063 Latvia
}

\author{
ARTICLE HISTORY \\ Compiled August 1, 2018
}

\begin{abstract}
The paper presents the results of detailed studies of the thermal expansion of $(1-$ $x) \mathrm{PbNi}_{1 / 3} \mathrm{Nb}_{2 / 3} \mathrm{O}_{3}-x \mathrm{PbTiO}_{3}$ solid solutions with $x=0-0$. The anomalous and lattice contributions to deformation and the thermal expansion coefficient are analysed and the mean square polarization $P_{d}$ is determined. The results obtained are discussed within the framework of the thermodynamic theory and the Landau 2-4-6 coefficients for solid solutions are estimated.
\end{abstract}

\section{KEYWORDS}

Thermal expansion; polarization; relaxors; perovskites

\section{Introduction}

In recent years, much attention has been paid to the study of oxide multicomponent mixed perovskites, which have a unique crystalline structure and unique physical properties, in particular, a huge dielectric constant, high piezoelectric and electrostriction coefficients, which makes them very promising for applications in various microelectronic devices. One of the most prominent representatives of such systems are (1$x) \mathrm{PNN}-x \mathrm{PT}$ solid solutions based on the relaxor $\mathrm{PbNi}_{1 / 3} \mathrm{Nb}_{2 / 3} \mathrm{O}_{3}(\mathrm{PNN})$ and the ferroelectric $\mathrm{PbTiO}_{3}$ (PT) [1, 2].

This paper presents the results of studies of the thermal expansion of ceramic samples of $(1-x) \mathrm{PNN}-x \mathrm{PT}(x=0-0.8)$ solid solutions in the temperature range from 100 to $750 \mathrm{~K}$ and the determination of polarization from dilatometric data.

\section{Experimental}

Samples were prepared at the Institute of Solid State Physics of the University of Latvia using conventional ceramic technology from the mixture of oxides: $\mathrm{PbO}, \mathrm{NiO}$, $\mathrm{Nb}_{2} \mathrm{O}_{5}$, and $\mathrm{TiO}_{2}$ [1]. The heat treatment conditions were optimized to prevent pyrochlore phase formation. The quality of the samples and their phase composition were checked by X-ray diffraction analysis at room temperature using a Bruker D8 ADVANCE X-ray powder diffractometer ( $\mathrm{Cu}-\alpha$ radiation). 
Thermal expansion was studied on ceramic samples with dimensions $L=5-15 \mathrm{~mm}$ in the temperature range $100-750 \mathrm{~K}$ on the induction dilatometer NETZSCH DIL-402C in a dynamic mode with heating and cooling rates of $2-3 \mathrm{~K} / \mathrm{min}$. The measurements were carried out by purging $(\sim 50 \mathrm{ml} / \mathrm{min})$ with dry helium.

\section{Results and discussion}

The temperature dependences of the thermal expansion coefficient $\beta$ and deformation $\Delta V / V$ of $(1-x) \mathrm{PNN}-x \mathrm{PT}$ compounds are presented in Fig. 1. It is found that the anomaly of the thermal expansion coefficient $\beta(T)$ at the transition from the cubic phase to the tetragonal phase (at $T_{0}$ ), characteristic of pure PT, remains rather sharp, but decreases considerably in amplitude in solid solutions $(1-x) \mathrm{PNN}-x \mathrm{PT}$ as $x$ decreases down to 0.35 . At concentrations $\mathrm{PT}$ smaller than the concentration of the morphotropic phase boundary $x_{M P B} \approx 0.30-0.35$, the anomaly is smeared out and becomes characteristic for ferroelectrics-relaxors with a minimum at $T_{m}$. In compositions with $x=0.80$ and 0.70 , as well as in pure $\mathrm{PbTiO}_{3}$, the phase transition remains a first-order transition, which is clearly seen from the results of measurements of the thermal expansion of these samples in the heating and cooling regimes. The hysteresis of the transition temperature was $3.6 \mathrm{~K}$ and $3.1 \mathrm{~K}$, respectively.
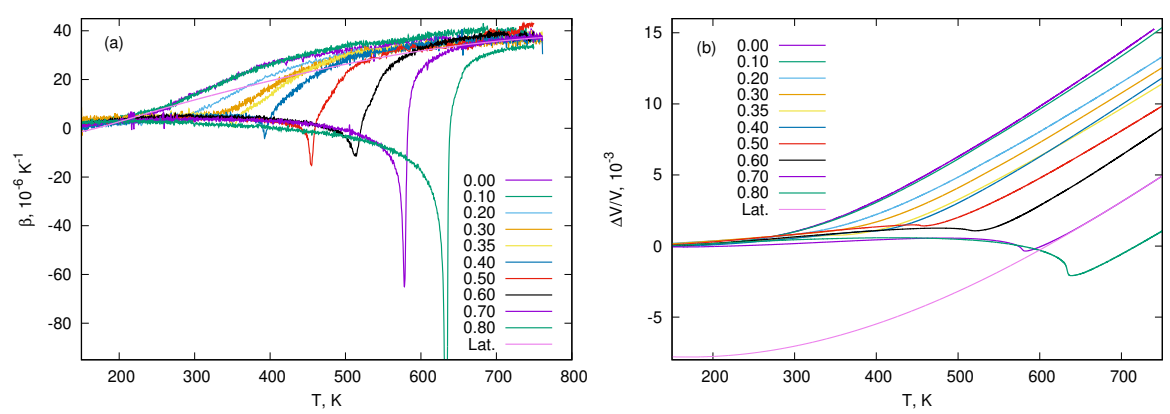

Figure 1. Temperature dependences of the volume thermal expansion coefficient(a) and bulk deformation (b) of solid solutions $(1-x) \mathrm{PNN}-x \mathrm{PT}$. Lat. - lattice (non-anomalous) component of deformation for the composition with $x=0.70$.

The anomalous volume deformation in lead titanate and other ferroelectrics with the perovskite structure $P m 3 m$ is mainly determined by the square of the spontaneous macroscopic polarization $\Delta V_{a n} / V=\left(Q_{11}+2 Q_{12}\right) P^{2}$ and, thus, can be used to estimate it. To extract the anomalous contribution to the deformation and to determine the mean-square polarization $P_{d}$, a correct description of the non-anomalous contribution to the volume deformation $\Delta V / V$ and the thermal expansion coefficient $\beta(T)$ are necessary.

The traditional method most often used in the literature [3], in which linear or volume deformation at high temperatures is approximated by linear dependences leads to overestimation the values of anomalous contribution to deformation, and the mean square polarization. In this paper we used another method taking into account the relationship between thermal expansion and heat capacity and its temperature dependence in the framework of the Debye model. The data was processed by a dependency $\beta(T)=a T+b C_{D}\left(T, \Theta_{D}\right)$, where $a, b$ and the Debye temperature $\Theta_{D}$ are fitting parameters. The results of processing the experimental data for the composition with $x=0.70$ are shown in Fig. 1(b). The temperature dependences of the mean-square polarization 
$P_{d}$ for all the compositions studied are shown in Fig. 2(a). For solid solutions, we used the same values of electrostriction coefficients as for pure $\mathrm{PbTiO}_{3}: Q_{11}=0.089 \mathrm{~m}^{4} / \mathrm{C}^{2}$, $Q_{12}=-0.026 \mathrm{~m}^{4} / \mathrm{C}^{2}[4]$.
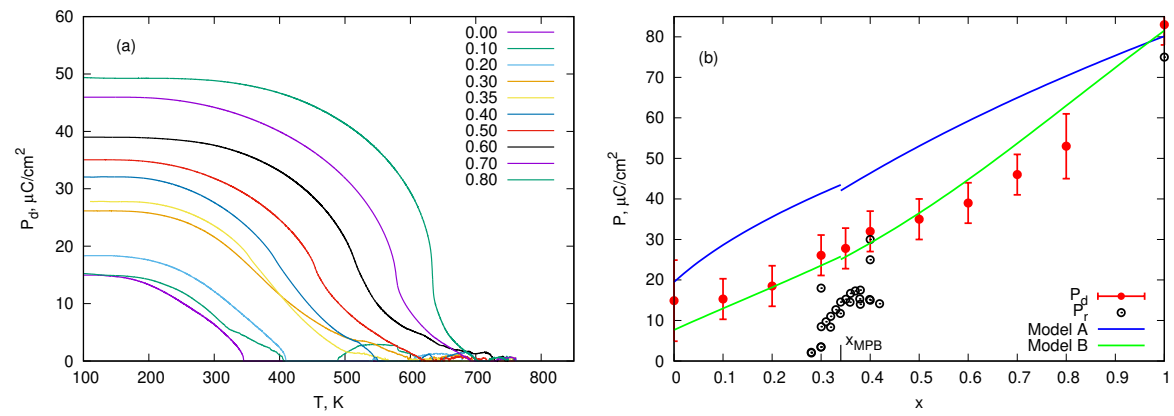

Figure 2. Temperature dependences of mean square polarization, determined from the thermal expansion of $(1-x) \mathrm{PNN}-x \mathrm{PT}$ solid solutions (a). Concentration dependences of the spontaneous polarization $\left(P_{r}\right)[5,2]$ and mean square polarization $P_{d}$, as well as the polarization calculated at $T=100 \mathrm{~K}$ from two sets of thermodynamic potential $\mathrm{PbTiO}_{3}$ (Model A [6], Model B [4]), for solid solutions $(1-x) \mathrm{PNN}-x \mathrm{PT}(\mathrm{b})$.

In mixed perovskites, the paraelectric cubic phase (point symmetry $m 3 m$ ) exists at high temperatures. In the traditional phase diagrams $T-x$ of these systems, the cubic phase transforms at low temperatures into ferroelectric rhombohedral (point symmetry $3 \mathrm{~m}$ ) and ferroelectric tetragonal (point symmetry $4 \mathrm{~mm}$ ) phases that have an almost vertical boundary (MPB).

A number of authors undertook a thermodynamic analysis of the morphotropic phase diagram using the traditional Ginzburg-Landau-Devonshire theory of phase transitions. Naturally, phenomenological theory requires experimental data, and therefore its predicative power is limited. Nevertheless, the qualitative features of the phase diagrams obtained with such an analysis, and the properties associated with them, may be of interest to researchers. It was shown that, in the first approximation, the appearance of an MPB corresponds to the condition that the symmetry of the free energy density varies from cubic to spherical [6].

Authors [6] considered the expansion of the thermodynamic potential to the sixth degree of polarization and split it into two parts - isotropic, not depending on the polarization direction, and anisotropic, depending on the direction of polarization $F=$ $F_{\text {iso }}+F_{\text {aniso }}$ :

$$
\begin{aligned}
F_{\text {iso }} & =\frac{1}{2} A_{1}\left(P_{1}^{2}+P_{2}^{2}+P_{3}^{2}\right)+\frac{1}{4} B_{1}\left(P_{1}^{2}+P_{2}^{2}+P_{3}^{2}\right)^{2}+\frac{1}{6} C_{1}\left(P_{1}^{2}+P_{2}^{2}+P_{3}^{2}\right)^{3}, \\
F_{\text {aniso }} & =\frac{1}{4} B_{2}\left(P_{1}^{4}+P_{2}^{4}+P_{3}^{4}\right)+\frac{1}{6} C_{2}\left(P_{1}^{6}+P_{2}^{6}+P_{3}^{6}\right)+\frac{1}{6} C_{3}\left(P_{1}^{2} P_{2}^{2} P_{3}^{2}\right) .
\end{aligned}
$$

To determine the expansion coefficients of the thermodynamic potential and their dependences on the concentration and, thus, to construct a concentration phase diagram and to calculate the polarization, deformation, and other properties of the system of solid solutions we need the coefficients for $\mathrm{PbTiO}_{3}$, the parameters of the triple point C-T-R $\left(x_{0}^{\prime}, T_{0}^{\prime}\right)$, the parameters of the tricritical point of phase transitions C-T $\left(x_{c r}^{T}, T_{c r}^{T}\right)$, as well as the temperature $T_{m}$ in relaxor [6].

Let's try to apply the approach developed by the authors [6], to our system $(1-$ $x) \mathrm{PNN}-x \mathrm{PT}$. The values of parameters obtained for solid solutions $(1-x) \mathrm{PNN}-x \mathrm{PT}$, determined from the two sets of coefficients of the thermodynamic potential for $\mathrm{PbTiO}_{3}$ 
are presented in Table 1, and the concentration dependence of polarization in Fig. 2(b).

Table 1. Parameters of the thermodynamic potential for PT and $(1-x) \mathrm{PNN}-x \mathrm{PT}$ solid solutions. $B_{2}(x, T)=$ $b_{0}\left(x_{0}^{\prime}-x\right)+b_{2}^{\prime}\left(T-T_{C}(x)\right)$

\begin{tabular}{lllll}
\hline Parameter & PT [6] & PNN-PT (Model A ) & PT [4] & PNN-PT (Model B) \\
\hline$T_{C}(x), \mathrm{K}$ & 748 & $748 x+153(1-x)$ & 752 & $752 x+153(1-x)$ \\
$A_{0}(x), 10^{5} \mathrm{Vm} / \mathrm{CK}$ & 7.95 & $7.95 x+1.85(1-x)$ & 7.6 & $7.60 x+1.99(1-x)$ \\
$B_{1}(x), 10^{8} \mathrm{Vm}^{5} / \mathrm{C}^{3}$ & 6.78 & $6.78 x+0.37(1-x)$ & -2.92 & $15 x+14.89(1-x)$ \\
$b_{0}, 10^{8} \mathrm{Vm}^{5} / \mathrm{C}^{3}$ & -9.66 & 14.63 & -17.92 & 27.15 \\
$b_{2}^{\prime}, 10^{5} \mathrm{Vm}^{5} / \mathrm{C}^{3}$ & -1.51 & -1.51 & - & - \\
$C_{1}, 10^{9} \mathrm{Vm}^{9} / \mathrm{C}^{5}$ & 1.39 & 1.39 & 1.22 & 1.22 \\
$C_{2}, 10^{8} \mathrm{Vm}^{9} / \mathrm{C}^{5}$ & 1.54 & 1.54 & 3.4 & 3.4 \\
$C_{3}, 10^{8} \mathrm{Vm}^{9} / \mathrm{C}^{5}$ & -4.63 & -4.63 & -295.2 & -295.2 \\
$\left(x_{0}^{\prime}, T_{0}^{\prime}\right)$ & - & $(0.34,355)$ & - & $(0.34,355)$ \\
$\left(x_{c r}^{T}, T_{c r}^{T}\right)$ & - & $(0.65,539.7)$ & - & $(0.65,539.7)$ \\
& & & &
\end{tabular}

\section{Conclusion}

Thus, as a result of the studies, anomalous behaviour of the deformation and coefficient of thermal expansion of solid solutions $(1-x) \mathrm{PNN}-x \mathrm{PT}$ was found and a concentration $T-x$ phase diagram was constructed. The results are analyzed in the framework of the thermodynamic theory of phase transitions and the coefficients of the expansion of the 2-4-6 thermodynamic Landau potential for a system of solid solutions $(1-x) \mathrm{PNN}-$ $x \mathrm{PT}$ are determined. The thermodynamic potential, taking into account the elastic and electrostriction components, makes it possible to calculate other thermodynamic properties, such as deformation, dielectric susceptibility, piezoelectric properties.

\section{References}

[1] Kallaev SN, Bormanis K, Omarov ZM, Mitarov RG, and Kalvane A: Thermal properties of ferroelectric $0.7 \mathrm{PbNi}_{1 / 3} \mathrm{Nb}_{2 / 3} \mathrm{O}_{3}-0.3 \mathrm{PbTiO}_{3}$ ceramics. Phase Transitions 2013;86: 886-892.

[2] Pan Zh, Chen J, Fan L, Wang Q, Liu L, Fang L, and Xing X: Both electric field and temperature independent behavior of piezoelectric property of $\mathrm{Pb}\left(\mathrm{Ni}_{1 / 3} \mathrm{Nb}_{2 / 3}\right) \mathrm{O}_{3}{ }^{-}$ $\mathrm{PbTiO}_{3}$. Mat. Res. Bull. 2015;61: 448 - 452.

[3] Li Y, Li J, Zhou Zh, Guo R, and Bhalla A: Thermal expansion behavior and polarization properties of lead-free ferroelectric potassium lithium tantalate niobate single crystals. Ceram. Internat. 2014;40: 1225-1228.

[4] Pertsev NA, Zembilgotov AG, and Tagantsev AK: Effect of Mechanical Boundary Conditions on Phase Diagrams of Epitaxial Ferroelectric Thin Films. Phys. Rev. Lett. 1998;80: 1988-1991.

[5] Lei $\mathrm{Ch}$, Chen $\mathrm{K}$, and Zhang $\mathrm{X}$ : Dielectric and ferroelectric properties of $\mathrm{Pb}\left(\mathrm{Ni}_{1 / 3} \mathrm{Nb}_{2 / 3}\right) \mathrm{O}_{3}-\mathrm{PbTiO}_{3}$ ferroelectic ceramic near the morphotropic phase boundary. Mat. Lett. 2002;54: 8-12. 
[6] Heitmann AA, and Rossetti GA: Thermodynamics of Ferroelectric Solid Solutions with Morphotropic Phase Boundaries. J. Am. Ceram. Soc. 2014;97: 1661-1685. 